\title{
Direct Insight into Ce-Silicates/Si-Nanoclusters Snowman-Like Janus Nanoparticles Formation in Ce-Doped $\mathrm{SiO}_{x}$ Thin Layers
}

\author{
Georges Beainy, ${ }^{\dagger}$ Jennifer Weimmerskirch-Aubatin, ${ }^{\ddagger}$ Mathieu Stoffel, ${ }^{\ddagger}$ Michel Vergnat, ${ }^{\ddagger}$ Hervé Rinnert, ${ }^{\ddagger}$ \\ Philippe Pareige, ${ }^{\dagger}$ and Etienne Talbot $^{*}{ }^{\dagger}$ \\ ${ }^{\dagger}$ Normandie Univ, UNIROUEN, INSA Rouen, CNRS, Groupe de Physique des Matériaux, 76000 Rouen, France \\ ${ }^{\ddagger}$ Université de Lorraine, UMR CNRS 7198, Institut Jean Lamour, BP 70239, 54506 Vandœuvre-lès-Nancy, France
}

ABSTRACT: The present work reports a nanoscale chemical and structural study on the influence of $\mathrm{Ce}$ content in $\mathrm{Ce}$ doped $\mathrm{SiO}_{1.5}$ thin films via atom probe tomography (APT). Using this technique, we can explore 3D mapping of the atomic distribution inside a material. Such an investigation is crucial to optimize the optical properties of Ce-doped $\mathrm{SiO}_{x}$ films. As a result, we clearly identify the influence of cerium on the phase separation process, on the silicon nanocrystal growth, and on the formation of cerium silicate nanoparticles occurring during annealing treatments. The observed nanoscale structure is correlated with the optical properties measured by room temperature photoluminescence spectroscopy thus leading to a comprehensive understanding of the properties of Ce-doped $\mathrm{SiO}_{1.5}$ thin films.

\section{INTRODUCTION}

Silicon photonics has been benefiting from an unprecedented boom over the last two decades. Several keystone components have been successfully demonstrated with remarkable performance, such as extremely low-loss waveguides, high bandwidth photodetectors, and ultrafast modulators. ${ }^{1}$ Despite these significant advances, the development of an efficient siliconbased light source has seriously lagged behind that of other photonic devices because of the low emission efficiency of bulk silicon. ${ }^{2}$ To progress toward this aim, several works were dedicated to the integration of III-V semiconductors or other materials such as $\mathrm{ZnO}$ with silicon because of the direct and wide band gaps of these compounds. ${ }^{3-5}$ However, because of the lattice and thermal mismatch between III-V materials and silicon, the integration remains a challenging issue. ${ }^{6}$ Another promising approach, which has attracted a lot of interest, is doping of silicon oxide thin films $\left(\mathrm{SiO}_{x}\right)$ with rare-earth elements. Depending on the rare-earth element considered, the emission can be tuned from ultraviolet (UV) to infrared. ${ }^{7-12}$ Up to now, efficient visible-light-emitting devices based on rareearth-doped metal-oxide-semiconductor (MOS) structures have been demonstrated. ${ }^{13,14}$ However, the solubility of rareearth ions in Si-based materials achieved so far is still relatively low $\left(10^{16}-10^{20} \mathrm{~cm}^{-3}\right){ }^{15}$ Moreover, for high rare earth ion concentrations, deterioration of luminescence efficiency is observed because of formation of ion clusters or secondary phases (e.g., oxides or optically inactive silicates). ${ }^{16,17}$ Such a concentration quenching presents a substantial barrier for further performance improvement of rare-earth-doped Si-based materials. Recently, it was shown that formation of rare-earth silicates (e.g., $\mathrm{Ce}_{2} \mathrm{Si}_{2} \mathrm{O}_{7}, \mathrm{Eu}_{2} \mathrm{SiO}_{5}, \mathrm{Er}_{2} \mathrm{Si}_{2} \mathrm{O}_{7}$ ) can enhance their photoluminescence efficiency. ${ }^{18-20}$ In these compounds, rareearth ions are optically active, and their solubility can reach $10^{22}$ $\mathrm{cm}^{-3} \cdot{ }^{20-22}$

Among rare-earth silicates, $\mathrm{Ce}_{2} \mathrm{Si}_{2} \mathrm{O}_{7}$ is a promising material for UV optoelectronic devices with an emission band close to that of $\mathrm{ZnO}$ and $\mathrm{GaN}$. The photoluminescence properties of $\mathrm{Ce}_{2} \mathrm{Si}_{2} \mathrm{O}_{7}$ have been investigated by several groups. ${ }^{18,19,23,24}$ Recently, we have reported the formation of Ce-silicate nanoclusters in substoichiometric 3 at. \% Ce-doped $\mathrm{SiO}_{1.5}$ thin films. Their formation is the result of a complex phase separation at the nanoscale between $\mathrm{Si}$ atoms in excess, $\mathrm{Ce}$ atoms, and the $\mathrm{SiO}_{2}$ matrix. ${ }^{25}$ However, only one particular $\mathrm{Ce}$ concentration (i.e., 3 at. \% Ce) was considered in this study. A systematic investigation of the effect of Ce concentration on the phase separation process could help to shed light into the complex nanoscale structural evolution of Ce-doped $\mathrm{SiO}_{1.5}$ thin films. In this paper, we perform an atomic scale investigation of Ce-doped $\mathrm{SiO}_{1.5}$ thin films as a function of $\mathrm{Ce}$ content by means of atom probe tomography. The observed nanoscale structure was then correlated with the optical properties studied by photoluminescence spectroscopy. The present paper leads to a comprehensive study of phase transformations occurring in Ce-doped $\mathrm{SiO}_{1.5}$ thin films and their relation with the optical properties. 


\section{EXPERIMENTAL SECTION}

Sample Preparation. Substoichiometric $\mathrm{SiO}_{1.5}$ thin films were deposited on top of $\mathrm{Si}(001)$ substrates by coevaporation of $\mathrm{SiO}$ powder from a thermal cell and $\mathrm{SiO}_{2}$ from an electron beam gun in an ultrahigh vacuum chamber. The film thickness was $200 \mathrm{~nm}$ for all the samples considered in this study. Cedoping was performed from a Knudsen cell heated around 1400 ${ }^{\circ} \mathrm{C}$. The Ce flux was monitored by a quartz microbalance, and the Ce content defined by $[\mathrm{Ce}] /([\mathrm{Si}]+[\mathrm{O}]+[\mathrm{Ce}])$ was varied between 0.7 at. $\%$ and 4 at. $\%$. The samples were postgrown annealed in a rapid thermal annealing furnace under flowing $\mathrm{N}_{2}$ for $5 \mathrm{~min}$ at $1100{ }^{\circ} \mathrm{C}$. At this particular annealing temperature, a rather strong Ce-related photoluminescence was already observed. ${ }^{25}$

Structural and Chemical Characterization. The 3D atomic scale characterization of the samples was performed using a laser-assisted wide-angle tomographic atom probe (LAWATAP-Cameca) instrument. Further information on atom probe tomography can be found elsewhere. ${ }^{26}$ In an atom probe tomography experiment, specimens must be needle-shaped having an end radius below $50 \mathrm{~nm}$. The preparation of such tips was achieved by employing lift-out and annular milling method, using a dual beam Zeiss NVision 40 focused ion beam scanning electron microscope (FIBSEM). ${ }^{27,28}$ To prevent any damage during the cutting and milling process, samples were covered by a $\sim 350 \mathrm{~nm}$ thick platinum layer before FIB processing. Photoluminescence (PL) measurements were performed at room temperature using the $325 \mathrm{~nm}$ line of a $\mathrm{He}-\mathrm{Cd}$ laser for the sample excitation. The PL signal was analyzed by a monochromator equipped with a 150 grooves/mm grating and by a cooled charge coupled device camera with a $300-1050 \mathrm{~nm}$ detection range.

\section{RESULTS AND DISCUSSION}

Photoluminescence Measurements. To investigate the impact of the Ce content on the optical properties, PL measurements on $\mathrm{SiO}_{1.5}$ films doped with a cerium concentration ranging from 0.7 to 4 at. \% were performed. All samples were annealed at $1100{ }^{\circ} \mathrm{C}$. The corresponding results are presented in Figure 1. Two emission bands are observed for $\mathrm{Ce}$ contents below 3 at. \%. The first one corresponds to a blue emission band centered at $450 \mathrm{~nm}$. The

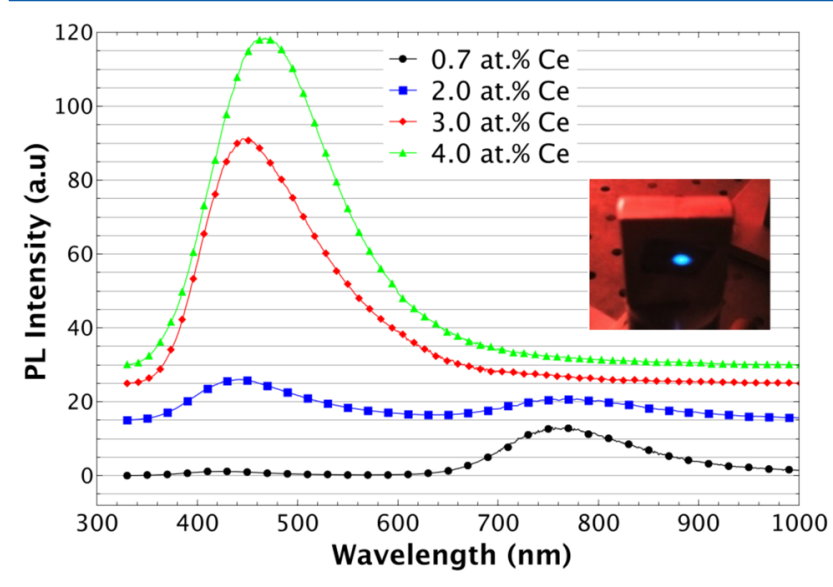

Figure 1. Room temperature (RT) PL spectra of Ce-doped $\mathrm{SiO}_{1.5}$ thin films annealed for $5 \mathrm{~min}$ at $1100{ }^{\circ} \mathrm{C}$. The Ce concentration is varied from 0.7 at. $\%$ to 4 at. $\%$. The inset shows the blue luminescence visible at $\mathrm{RT}$ with the naked eyes. emission can be attributed to the electric dipolar allowed $5 \mathrm{~d} \rightarrow$ 4f transition of $\mathrm{Ce}^{3+}$ ions. The second one, in the near-infrared centered at $750 \mathrm{~nm}$, is due to radiative recombinations between electrons and holes confined in silicon nanoclusters (Si-NCs). ${ }^{29}$

The existence of Si-NCs in the layers and their optical signature is expected. Indeed, it is well-known that the presence of $\mathrm{Si}$ atoms in excess in substoichiometric $\mathrm{SiO}_{x}$ thin films can result in the formation of Si nanocrystals when the films are annealed at sufficiently high temperatures. ${ }^{30}$ When the Ce content in the films increases, the Si-NCs related luminescence decreases while simultaneously that of Ce increases, suggesting an influence of the $\mathrm{Ce}$ on the growth and on the optical properties of Si-NCs. For the 0.7 and 2 at. \% Ce-doped samples, the $\mathrm{Ce}^{3+}$ related luminescence is weak. Yet, a significant improvement was observed for the 3 and 4 at. \% Ce-doped layers, indicating that the Ce-related luminescence does not increase linearly with the Ce concentration. The Cerelated luminescence from the 3 at. $\%$ and 4 at. \% doped samples is strong and can be even seen with the naked eyes (see inset of Figure 1). Moreover, we can note a red-shift of the Cerelated luminescence when the Ce concentration increases to 3 and $4 \%$. This may possibly be due to a modification of the local environment of $\mathrm{Ce}^{3+}$ ions. To elucidate the mechanism underlying the evolution of PL intensities and the quenching of the Si-NCs related PL for high Ce contents (between 2 and 3 at. \%), the nanoscale structure of the samples was investigated by atom probe tomography.

Structural Evolution. All the samples were analyzed by atom probe tomography. Figure 2 shows the obtained 3D maps for which cluster identification was performed on $\mathrm{Si}$ and $\mathrm{Ce}$ to detect the presence or absence of precipitates in the samples using the GPM's data treatment code. In all samples, pure Si nanoclusters (pink) and Ce-rich clusters (blue) were identified. Moreover, we observe that Ce-rich clusters are always localized at the vicinity of a Si-NC whatever the Ce concentration. The sample doped with 0.7 at. \% of Ce presents a high number density of Si nanoparticles (Figure 2a) which are well separated from Ce nanoclusters. For the 2 at. \% Ce doped layer (Figure $2 \mathrm{~b}$ ), the number density of small, isolated Si-NCs strongly decreases. Meanwhile, the population of Ce-rich clusters, as well as that of large, coalesced $\mathrm{Si}$ nanoparticles increases. Further increase in the Ce content up to 3 at. \% and 4 at. \% (Figure $2 \mathrm{c}$ and $2 \mathrm{~d}$, respectively) leads to an additional decrease in the number density of $\mathrm{Si}$ nanoparticles. Furthermore, all $\mathrm{Si}$ nanoparticles identified in the volume for 3 and 4 at. \% of Ce are linked to Ce-rich nanoclusters. The impact of Ce content on the growth of Si nanoparticles is clearly demonstrated. The $3 \mathrm{D}$ reconstructions reveal the existence of two populations of $\mathrm{Si}$ nanoparticles: small ones, isolated in the matrix, or larger ones bounded to Ce-rich nanoclusters. The isolated Si-NCs appear only for low Ce contents ( 0.7 and 2 at. \%), whereas, for the 3 and 4 at. \% Ce-doped layers, all the silicon nanoparticles are located in the immediate proximity of Ce-rich precipitates. The modification of the $\mathrm{Si}$ nanoparticle growth mechanism in the layers is strongly correlated with the increase of the Ce content. As it can be seen in Figure 2, their size and number density are clearly strongly impacted. Subsequently, we will look to the quantification of these parameters in order to better understand the mechanisms governing this process. The nanoparticles are spherical, and the observed elongated shape is due to local magnification effects during APT experiment analysis as previously demonstrated on similar systems. ${ }^{25,31}$ 
(a)

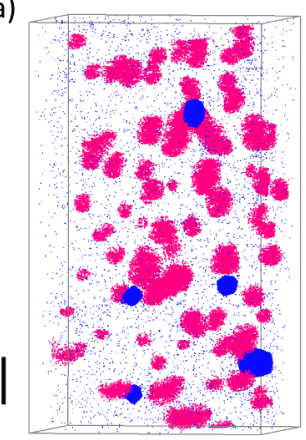

(b)

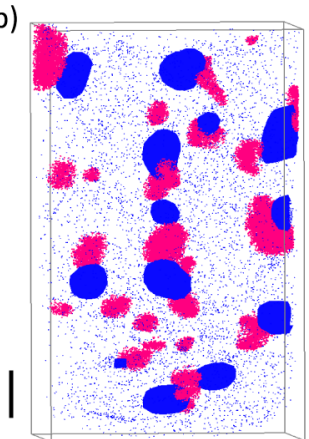

(c)

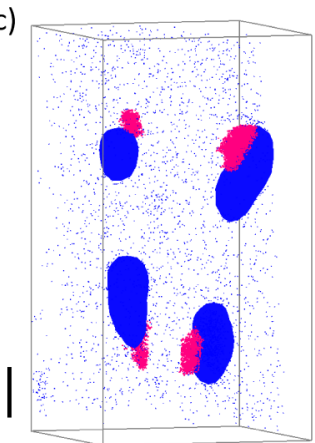

(d)

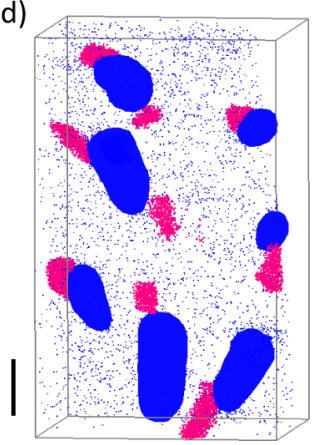

Figure 2. Distribution of Si nanoparticles (pink) and Ce-rich clusters (blue) in the analyzed volumes obtained by atom probe tomography for Cedoped $\mathrm{SiO}_{1.5}$ thin films: (a) 0.7 at. \% Ce, (b) 2 at. \% Ce, (c) 3 at. \% Ce, and (d) 4 at. \% Ce. Isolated blue dots correspond to free Ce atoms in the $\mathrm{SiO}_{2}$ matrix. Scale bar is $5 \mathrm{~nm}$.

Composition Measurements. First, we will focus on the identification of the phases existing in the samples. In all the samples, the matrix composition corresponds to $\mathrm{SiO}_{2}$ comprising each time in addition a fraction of isolated $\mathrm{Ce}$ atoms as observed in Figure 2 (identified by isolated blue dots). All other $\mathrm{Ce}$ ions are located in the Ce-rich clusters. The average compositions of the Ce-rich particles are presented on the ternary $\mathrm{O}-\mathrm{Ce}-\mathrm{Si}$ phase diagram shown in Figure 3. For

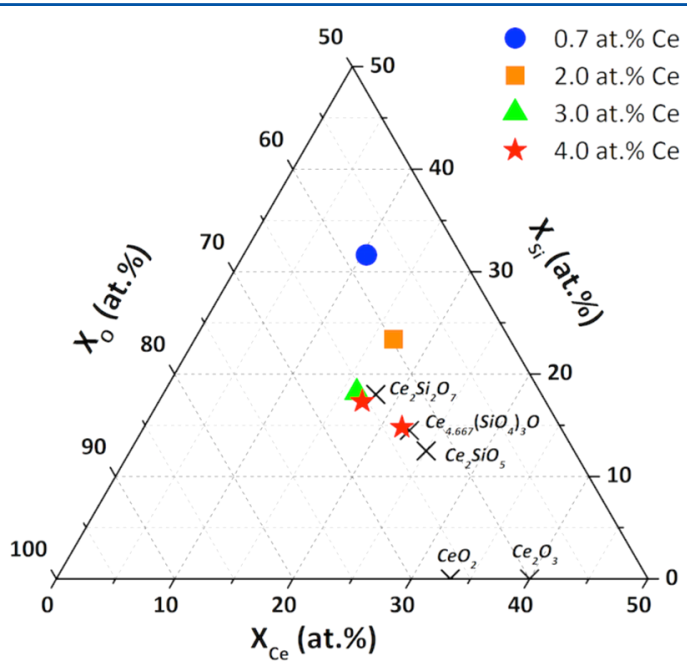

Figure 3. Mean composition measurements of Ce-rich clusters determined from APT analysis for the different Ce concentrations plotted on the ternary $\mathrm{O}-\mathrm{Ce}-\mathrm{Si}$ phase diagram.

the 0.7 and 2 at. \% Ce-doped samples, the obtained composition does not match with any stoichiometry of a defined cerium silicate compound. A stable cerium silicate phase of stoichiometry $\mathrm{Ce}_{2} \mathrm{Si}_{2} \mathrm{O}_{7}$ is identified for the 3 at. \% Cedoped sample. Finally, for the 4 at. \% Ce-doped sample, the measurements give evidence of the existence of two compounds with different stoichiometry: $\mathrm{Ce}_{2} \mathrm{Si}_{2} \mathrm{O}_{7}$ and $\mathrm{Ce}_{4.667}\left(\mathrm{SiO}_{4}\right)_{3} \mathrm{O}$. Their amount in the studied volume corresponds to $43 \%$ and $57 \%$, respectively, for $\mathrm{Ce}_{2} \mathrm{Si}_{2} \mathrm{O}_{7}$ and $\mathrm{Ce}_{4.667}\left(\mathrm{SiO}_{4}\right)_{3} \mathrm{O}$. The coexistence of these two compounds has already been mentioned in the literature. ${ }^{19,24}$ High-temperature annealing of cerium oxide layers leads to the formation of cerium silicates at the interface with the silica or silicon substrates. In this contribution, we evidenced the formation of cerium silicates in the form of nanoparticles in substoichiometric Ce-doped $\mathrm{SiO}_{1.5}$ films. Cerium silicates can exist in various polymorphs. ${ }^{32}$ The identification of such polymorphs was, however, not possible and appears to be clearly beyond the scope of the present paper. As mentioned previously, all $\mathrm{Ce}$ atoms did not precipitate into clusters, and a small quantity remains diluted in the silica matrix. This quantity allows us to determine the solubility limit of $\mathrm{Ce}$ in silica or silicon-rich silicon oxide films assuming that coalescence stage is reached. On the basis of the measurement of the Ce atomic fraction in the silica matrix, the solubility limit of $\mathrm{Ce}$ in $\mathrm{SiO}_{2}$ can be estimated to be $0.8 \pm 0.2 \%$ (i.e., $5.3 \pm 1.3 \times 10^{20} \mathrm{~cm}^{-3}$ ) for samples annealed at $1100{ }^{\circ} \mathrm{C}$. Finally, all Si atoms in excess have precipitated into pure $\mathrm{Si}$ nanoparticles or are located in the Ce-rich clusters.

Morphological Information. To quantitatively evaluate the influence of $\mathrm{Ce}$ on the growth of silicon nanoparticles, we have measured the number density and the radius of both $\mathrm{Ce}$ nanoclusters and Si-NCs (isolated or linked to Ce nanoclusters).

Figure 4a shows the evolution of the density of nanoparticles with the nominal Ce concentration in the films. The number density of the isolated Si-NCs monotonously decreases with the Ce content until $3 \%$. The evolution of the number density of linked Si-nanoparticles (shown as triangle in Figure 4a) reveals that this value is nearly constant indicating a strong decrease of the number density of isolated Si-NCs. This highlights the effect of $\mathrm{Ce}$ on the growth mechanism of $\mathrm{Si}$ nanoparticles that does not take place as in undoped $\mathrm{SiO}_{x}$ films. Indeed, as the number of $\mathrm{Si}$ atoms in excess and the annealing temperature are constant, the density of Si-NCs should be constant if the Ce atoms do not influence the Si-NCs growth mechanism. The Ce-rich clusters number density increases substantially as the Ce content increases from 0.7 to 2 at. \%. This evolution can be attributed to the increase of the $\mathrm{Ce}$ concentration which induces more inhomogeneity in the sample and germination sites but without formation of any stable compound. However, for the 3 at. \% and 4 at. \% doped samples, the formation of cerium silicates induces a decrease of the Ce-rich particle number density.

Figure $4 \mathrm{~b}$ shows the evolution of the mean diameter of both Ce-rich and Si nanoclusters with the Ce content in the films. In this figure, we divide the concentration effect in three regimes: regime (I) corresponding to Ce concentrations up to 2 at. \%, where isolated Si-NCs are present, regime (II) corresponding to Ce contents between 2 and 3 at. \%, and finally, regime (III) corresponding to Ce contents beyond 3 at. \% where only linked Si-NCs can be observed. 
a)

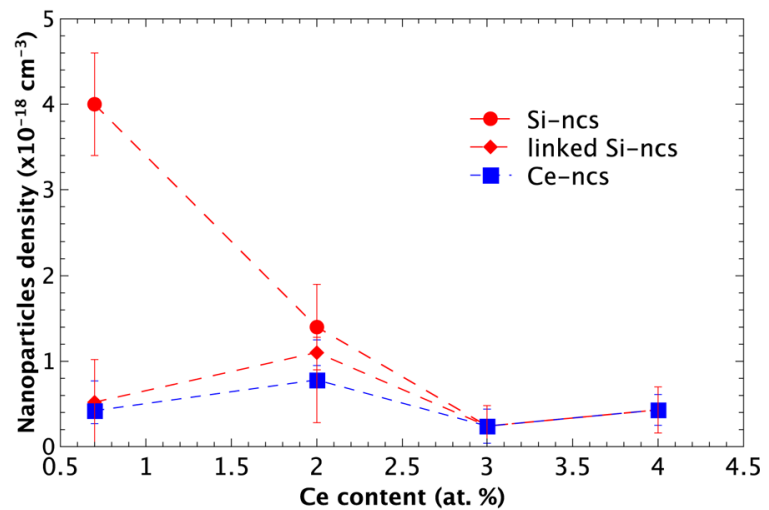

b)

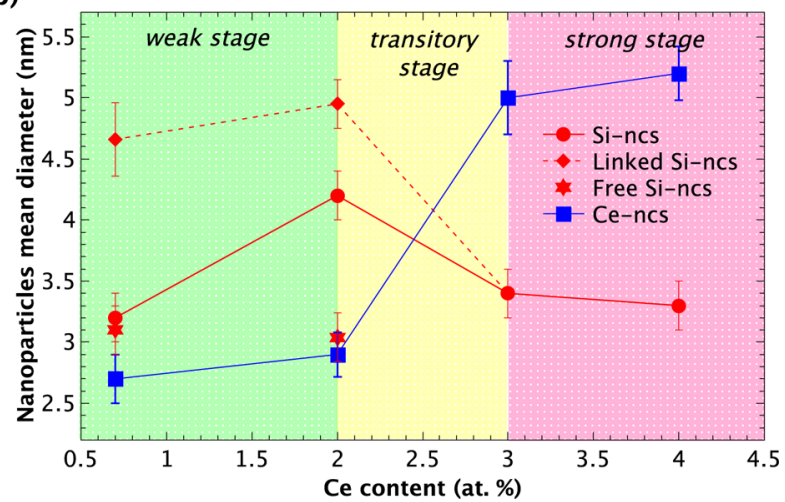

Figure 4. (a) Density of nanoparticles (silicon or Ce-rich) as a function of the Ce content. (b) Mean diameter of nanoparticles (silicon or Ce-rich) as a function of the Ce content.

The mean size of the Ce-rich clusters does not change in either regime I or III. However, their size increases by nearly a factor of 2 when the Ce content increases from 2 at. \% to 3 at. $\%$. This evolution may be related to an increasing consumption of $\mathrm{Ce}$ atoms (as evidenced by the increase of the mean $\mathrm{Ce}$ composition of Ce-rich nanoclusters in Figure 3 and by the decrease of the Ce-rich nanocluster density shown in Figure 4a) leading eventually to the formation of cerium silicate nanoparticles.
Regarding the diameter of Si nanoparticles (Figure $4 b$ ), the average size measured for the sample containing 0.7 at. \% $\mathrm{Ce}$ is $3.2 \pm 0.2 \mathrm{~nm}$. For this sample, $90 \%$ of Si-NCs are isolated in the matrix. These free particles have an average size of $3.1 \pm 0.2$ $\mathrm{nm}$. In contrast, the other $10 \%$ of Si-NCs (linked to Ce-NCs) have an average size of $4.6 \pm 0.3 \mathrm{~nm}$, which is larger than the size of the free particles. For a concentration of 2 at. \% Ce, we observe an increase in the $\mathrm{Si}-\mathrm{NCs}$ average size. The mean size of isolated Si-NCs is $3.0 \pm 0.2 \mathrm{~nm}$. This value is comparable with that measured for the sample doped with 0.7 at. \% $\mathrm{Ce}$ in which $90 \%$ of Si-NCs were free. Thus, the free particles are not affected by the presence of Ce. Linked Si nanoparticles have a mean size of $4.9 \pm 0.2 \mathrm{~nm}$. The population of linked Si-NCs increases from $10 \%$ for the 0.7 at. \% Ce-doped sample to $55 \%$ for the 2 at. \% Ce-doped sample. This increase in the proportion of linked $\mathrm{Si}-\mathrm{NCs}$ is therefore responsible for the increase in the average size of the sample doped with 2 at. \% Ce. In samples doped with 3 at. \% Ce and 4 at. \% Ce, all Si$\mathrm{NCs}$ are linked to cerium silicate nanoparticles, and their average size decreases to $3.4 \pm 0.2 \mathrm{~nm}$. As the Ce content increases, an increasing number of stoichiometric cerium silicate nanoparticles are formed thereby consuming $\mathrm{Si}$ atoms in excess. As a result, less $\mathrm{Si}$ atoms are available to form pure SiNCs. This can be explained by the fact that with the increase of the amount of $\mathrm{Ce}$, stoichiometric cerium silicates are formed which consume more $\mathrm{Si}$ atoms, and less $\mathrm{Si}$ atoms are ejected during the phase separation, and therefore, less Si excess is available to form Si-NCs. ${ }^{25,33}$ For high Ce concentrations (3 at. $\%)$, areas rich in $\mathrm{Ce}, \mathrm{O}$, and $\mathrm{Si}$ are initially formed during the deposition process. Then, $\mathrm{Si}$ nanoparticles are created through a phase separation mechanism from $\mathrm{Ce}-\mathrm{Si}-\mathrm{O}$ rich particles to Si-NCs and cerium silicate. Therefore, no classical germination growth of free Si-NCs is observed up to 2 at. \% of Ce.

Precipitation Mechanism. To explain the growth of cerium silicate nanoparticles and the evolution of linked versus free Si-NCs, we propose a precipitation mechanism as a function of annealing temperature and Ce content. We have previously reported a phase separation occurring at the nanoscale between pure Si-NCs and cerium silicate NCs in a $\mathrm{SiO}_{1.5}$ film doped with 3 at. \% Ce. ${ }^{25}$

The diagram shown in Figure 5 summarizes the morphological and chemical changes observed by atom probe

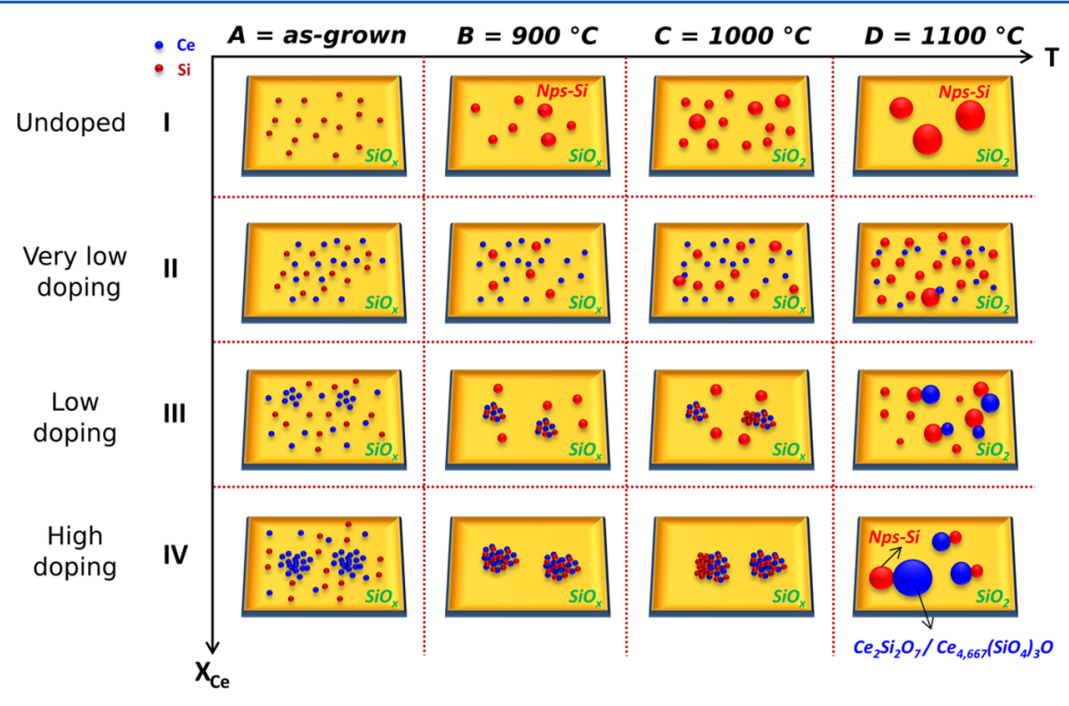

Figure 5. Schematic representation of the precipitation mechanism in Ce-doped $\mathrm{SiO}_{1.5}$ thin films as a function of temperature and Ce doping level. 
tomography experiments performed on Ce-doped $\mathrm{SiO}_{1.5}$ thin films as a function of annealing temperature and Ce content. In this diagram, we have divided the influence of Ce content on the diffusion and precipitation of $\mathrm{Si}$ and cerium silicates with annealing temperature in four different regimes.

Regime I. Undoped $\mathrm{SiO}_{x}$ Films. For the as-deposited sample (I.A), silicon atoms can be either distributed homogeneously or not, depending on the incorporated Si excess. ${ }^{34,35}$ The thermal annealing activates the phase separation between $\mathrm{Si}$ and $\mathrm{SiO}_{2}$. During the early stages of annealing (I.B), germination and growth of pure $\mathrm{Si}$ nanoparticles occur, and the matrix still contains $\mathrm{Si}$ atoms in excess. During the precipitation process (I.C), the excess of $\mathrm{Si}$ is consumed gradually until that oversaturation is completely consumed. When all $\mathrm{Si}$ atoms in excess are precipitated, the precipitation process is complete, and the matrix is composed of $\mathrm{SiO}_{2}$. Moreover, the growth rate and diffusion coefficient strongly depends on the elaboration conditions and the amount of $\mathrm{Si}$ atoms in excess. In the last stage (I.D), coalescence takes place in the films leading to an increased particle size and to a reduced density.

Regime II. Low-Doped Cerium Films ( $X_{C e}<$ Ce Solubility Limit in $\mathrm{SiO}_{2}$ ). The $\mathrm{Ce}$ atoms do not significantly affect the precipitation of the Si nanoparticles. Indeed, in the case of the 0.7 at. \% Ce-doped film, $90 \%$ of the Si nanoparticles remain isolated. Moreover, their mean size and density are compatible with those measured on undoped $\mathrm{SiO}_{x}$ films. Whatever the annealing temperature, the formation of $\mathrm{Si}$ nanoparticles is almost identical to that of regime I, and the majority of cerium ions remains diluted in the matrix. In this case, segregation of Ce is not observed.

Regime III. Intermediate-Doped Cerium Films (Ce Solubility Limit in $\mathrm{SiO}_{2}<X_{\mathrm{Ce}}<\sim 2$ at. \%). The formation of small Ce seeds is observed while the distribution of $\mathrm{Si}$ atoms remains homogeneous in as-grown films (III.A). ${ }^{25}$ During annealing, $\mathrm{Si}$ atoms diffuse toward the $\mathrm{Ce}$ seeds to form nonstable $\mathrm{Si}-\mathrm{O}-\mathrm{Ce}$ rich clusters. Meanwhile, the diffusing $\mathrm{Si}$ atoms also lead to the formation of $\mathrm{Si}$ nanoparticles in a conventional manner (III.B). Two mechanisms thus occur simultaneously: a phase separation between $\mathrm{Si}$ and $\mathrm{SiO}_{2}$ and an accumulation of $\mathrm{Ce}$ atoms exceeding locally the segregation of cerium in excess compared to the solubility limit. In this regime, the Ce content in the $\mathrm{Si}-\mathrm{O}-\mathrm{Ce}$ rich clusters is still too low to form a stoichiometric cerium silicate phase. There is, therefore, a creation of local inhomogeneity of Ce with elaboration and slight change in the first stages of annealing. On the other hand, $\mathrm{SiO}_{x}$ phase separation tends to eject $\mathrm{Si}$ from $\mathrm{SiO}_{2}$. The $\mathrm{Si}$ atoms in excess enter either into pure $\mathrm{Si}$ nanoparticles or into $\mathrm{Si}-\mathrm{O}-$ $\mathrm{Ce}$ clusters. On the basis of our observations, it appears that $\mathrm{Si}$ atoms in excess located close to Ce-rich clusters ejected Si that forms silicon nanoparticles and areas rich in $\mathrm{Si}-\mathrm{Ce}-\mathrm{O}$. Silicon in excess near Ce-rich zones go to those areas rather than forming isolated and pure Si nanoparticles.

Regime IV. Case of Highly Doped Cerium Films $\left(X_{C e}>3\right.$ at. $\%)$. In this regime, the distribution of $\mathrm{Ce}$ atoms is increasingly inhomogeneous while the distribution of $\mathrm{Si}$ atoms remains homogeneous in as-grown films. During annealing, the $\mathrm{Si}$ atoms in excess will mainly diffuse toward the Ce-rich seeds (IV.B). For annealing temperatures larger than $900{ }^{\circ} \mathrm{C}, \mathrm{Si}$ atoms start to be ejected from the $\mathrm{Si}-\mathrm{Ce}-\mathrm{O}$ clusters, and eventually, pure Si-nanocrystals start to form. ${ }^{25}$ For annealing temperatures close to $1100{ }^{\circ} \mathrm{C}$, the thermal budget allows the precipitation of cerium silicate nanoparticles to minimize the free energy of the system. The $\mathrm{Si}-\mathrm{Ce}-\mathrm{O}$ clusters thus completely dissociate into pure Si-NCs, and cerium silicate nanoparticles thus lead to the observation of snowman-like nanoparticles. The system tends to form a cerium silicate phase to minimize its energy. With additional thermal contributions, the system energy is changed by increasing the degree of disorder or entropy. Once the matrix consisting of $\mathrm{SiO}_{2}+\mathrm{Ce}$ is diluted, a phase separation at the nanoscale is thus observed between $\mathrm{Si}$ and $\mathrm{Si}-\mathrm{Ce}-\mathrm{O}$ (IV.C). Si atoms are ejected from these areas. At higher temperature, the energy supply is sufficient to complete this phase separation. Rich areas dissociate completely into pure $\mathrm{Si}$ nanoparticles and cerium silicate phases. The lack of thermodynamic data on cerium silicates limits the explanation of the observed reaction and, therefore, which silicate is the most energetically favorable phase or not. The observed nanostructure is remarkable. $\mathrm{Si}$ nanoparticles grow on the surface of cerium silicates. This nanostructure which has been identified in the literature as snowman-like nanoparticles was mainly observed during synthesis of colloidal nanoparticles. ${ }^{36,37}$ To our knowledge, the demonstration of such a configuration in thin films has never been reported.

The proposed precipitation mechanism has been extracted by varying the Ce content at a constant silicon excess of $10 \%$. On the basis of our previous work on Er-doped silicon-rich silica with less silicon excess, ${ }^{17}$ we observed a similar formation of both pure Si-NCs and Er-rich nanoparticles, highlighting that the precipitation mechanism can be generalized to other $\mathrm{RE}^{3+}$ ions. However, this assumption should be confirmed by a systematic investigation of highly doped nanostructures and for high-annealing temperatures. On the other hand, the influence of silicon excess on the phase separation process can be divided into two stages: (a) first, at low annealing temperature and depending on the RE dopant content, a portion or all of the silicon atoms in excess are consumed to form $\mathrm{RE}-\mathrm{Si}-\mathrm{O}$ rich clusters. (b) Second, at high-annealing temperature, the RE$\mathrm{Si}-\mathrm{O}$ rich clusters will be decomposed into stable RE-silicate phases and pure Si-NCs. ${ }^{25}$ Therefore, the density and the composition of the formed nanoparticles are highly influenced by the silicon excess and the RE content.

Correlation between the Nanostructure and the Optical Properties. The nanostructures observed previously by atom probe tomography help us to understand the changes in the optical properties shown in Figure 1. Indeed, the weak Ce-related luminescence observed for the samples doped with 0.7 at. $\%$ and 2 at. \% Ce originates from isolated $\mathrm{Ce}^{3+}$ ions. Indeed, for the sample doped with 0.7 at. \% Ce showing the lowest Ce-related luminescence, most of the Ce ions (95\%) remain isolated in the matrix. For the sample doped with 2 at. $\% \mathrm{Ce}$, the Ce-related luminescence increases and further slightly shifts to larger wavelengths. This indicates that some Ce ions must have a different chemical environment. Indeed, we found that a small amount of cerium silicate nanoparticles are already present in the sample doped with 2 at. \% Ce in addition to the isolated $\mathrm{Ce}^{3+}$ ions. This can be explained by the precipitation and the formation of a small amount of Ce silicate $\left(\mathrm{Ce}_{2} \mathrm{Si}_{2} \mathrm{O}_{7}\right)(20 \%)$. For higher $\mathrm{Ce}$ contents, the Ce-related luminescence increases dramatically while a clear shift is observed to higher wavelengths indicating that an increasing number of $\mathrm{Ce}$ atoms participate in the formation of optically active cerium silicates nanoparticles. In contrast, the Si-NCs related luminescence decreases gradually as the Ce content increases in the films. This may possibly be related to an increasing size of the Si-NCs which could lead to a loss of the quantum confinement. However, we have shown that the Si- 
NC size remains well below $5 \mathrm{~nm}$ (Figure $4 \mathrm{~b}$ ) thus allowing the exclusion of this first hypothesis. We thus explain the observed evolution by the decreasing density of free Si-NCs (Figure 4a) for high Ce contents. For the samples doped with 3 at. $\%$ and 4 at. \% Ce, all Si-NCs are indeed linked to the cerium silicate nanoparticles (Figure 2). We expect that the close vicinity of cerium silicate nanoparticles will provide nonradiative recombination pathways for excitons confined in pure Si-NCs.

\section{CONCLUSIONS}

To summarize, our investigations helped to shed light into the complex pathways leading to precipitation and phase separation in Ce-doped $\mathrm{SiO}_{1.5}$ thin films. Thanks to atom probe tomography, which allows a $3 \mathrm{D}$ reconstruction of the matter at the atomic scale, we provide evidence of the formation of snowman-like nanostructures consisting of pure Si-NCs linked to cerium silicate nanoparticles. The influence of $\mathrm{Ce}$ content on the formation of Si-NCs and cerium silicate nanoparticles is discussed. On the basis of our observations, we were able to propose a precipitation mechanism in Ce-doped $\mathrm{SiO}_{1.5}$ thin films as a function of both annealing temperature and $\mathrm{Ce}$ content. This allowed us to get a direct comprehensive view of the optical properties of the samples investigated by roomtemperature photoluminescence. Such a deep correlation between optical properties and structure at the nanoscale represents an important step toward the realization of future $\mathrm{Si}$ based light-emitting devices.

\section{ACKNOWLEDGMENTS}

The authors would like to acknowledge the financial support of Georges Beainy from the Région Haute-Normandie. The authors acknowledge the METSA network.

\section{REFERENCES}

(1) Reed, G. T.; Mashanovich, G.; Gardes, F. Y.; Thomson, D. J. Silicon Optical Modulators. Nat. Photonics 2010, 4, 518-526.

(2) Hirschman, K. D.; Tsybeskov, L.; Duttagupta, S. P.; Fauchet, P. M. Silicon-Based Visible Light-Emitting Devices Integrated into Microelectronic Circuits. Nature 1996, 384, 338-341.

(3) Ra, Y.-H.; Navamathavan, R.; Park, J.-H.; Lee, C.-R. High-Quality Uniaxial $\operatorname{In}_{x} \mathrm{Ga}_{1 x} \mathrm{~N} / \mathrm{GaN}$ Multiple Quantum Well (MQW) Nanowires (NWs) on $\mathrm{Si}(111)$ Grown by Metal-Organic Chemical Vapor
Deposition (MOCVD) and Light-Emitting Diode (LED) Fabrication. ACS Appl. Mater. Interfaces 2013, 5, 2111-2117.

(4) Nguyen, H. P. T.; Zhang, S.; Cui, K.; Han, X.; Fathololoumi, S.; Couillard, M.; Botton, G. A.; Mi, Z. p-Type Modulation Doped InGaN/GaN Dot-in-a-Wire White-Light-Emitting Diodes Monolithically Grown on $\mathrm{Si}(111)$. Nano Lett. 2011, 11, 1919-1924.

(5) Hsieh, Y.-P.; Chen, H.-Y.; Lin, M.-Z.; Shiu, S.-C.; Hofmann, M.; Chern, M.-Y.; Jia, X.; Yang, Y.-J.; Chang, H.-J.; Huang, H.-M.; et al. Electroluminescence from $\mathrm{ZnO} / \mathrm{Si}$-Nanotips Light-Emitting Diodes. Nano Lett. 2009, 9, 1839-1843.

(6) Trivino, N. V.; Dharanipathy, U.; Carlin, J.-F.; Diao, Z.; Houdré, R.; Grandjean, N. Integrated Photonics on Silicon with Wide Bandgap GaN Semiconductor. Appl. Phys. Lett. 2013, 102, 081120.

(7) Izeddin, I.; Moskalenko, A. S.; Yassievich, I. N.; Fujii, M.; Gregorkiewicz, T. Nanosecond Dynamics of the Near-Infrared Photoluminescence of Er-Doped $\mathrm{SiO}_{2}$ Sensitized with Si Nanocrystals. Phys. Rev. Lett. 2006, 97, 207401.

(8) Steveler, E.; Rinnert, H.; Vergnat, M. Direct and Indirect Excitation of $\mathrm{Nd}^{3+}$ Ions Sensitized by Si Nanocrystals Embedded in a $\mathrm{SiO}_{2}$ Thin Film. J. Appl. Phys. 2011, 110, 113518.

(9) Li, J.; Zalloum, O.; Roschuk, T.; Heng, C.; Wojcik, J.; Mascher, P. Light Emission from Rare-Earth Doped Silicon Nanostructures. Adv. Opt. Technol. 2008, 2008, 1.

(10) Debieu, O.; Breard, D.; Podhorodecki, A.; Zatryb, G.; Misiewicz, J.; Labbe, C.; Cardin, J.; Gourbilleau, F. Effect of Annealing and Nd Concentration on the Photoluminescence of $\mathrm{Nd}^{3+}$ Ions Coupled with Silicon Nanoparticles. J. Appl. Phys. 2010, 108, 113114.

(11) Podhorodecki, A.; Golacki, L.; Zatryb, G.; Misiewicz, J.; Wang, J.; Jadwisienczak, W.; Fedus, K.; Wolcik, J.; Wilson, P. Excitation Mechanism and Thermal Emission Quenching of $\mathrm{Tb}$ Ions in Silicon Rich Silicon Oxide Thin Films Grown by Plasma-Enhanced Chemical Vapour Deposition-Do We Need Silicon Nanoclusters? J. Appl. Phys. 2014, 115, 143150 .

(12) Rebohle, L.; Berencen, Y.; Wutzler, R.; Braun, M.; Hiller, D.; Ramirez, J.; Garrido, B.; Helm, M.; Skorupa, W. The Electroluminescence Mechanism of $\mathrm{Er}^{3+}$ in Different Silicon Oxide and Silicon Nitride Environments. J. Appl. Phys. 2014, 116, 123104.

(13) Sun, J. M.; Skorupa, W.; Dekorsy, T.; Helm, M.; Rebohle, L.; Gebel, T. Efficient Ultraviolet Electroluminescence from a GdImplanted Silicon Metal-Oxide-Semiconductor Device. Appl. Phys. Lett. 2004, 85, 3387-3389.

(14) Sun, J. M.; Skorupa, W.; Dekorsy, T.; Helm, M.; Rebohle, L.; Gebel, T. Bright Green Electroluminescence from $\mathrm{Tb}^{3+}$ in Silicon Metal-Oxide-Semiconductor Devices. J. Appl. Phys. 2005, 97, 123513.

(15) Polman, A.; van den Hoven, G. N.; Custer, J. S.; Shin, J. H.; Serna, R.; Alkemade, P. F. A. Erbium in Crystal Silicon: Optical Activation, Excitation, and Concentration Limits. J. Appl. Phys. 1995, $77,1256-1262$.

(16) Thogersen, A.; Mayndi, J.; Vines, L.; nd A. Olsen, M. S.; Diplas, S.; Mitome, M.; Bando, Y. The Formation of Er-Oxide Nanoclusters in $\mathrm{SiO}_{2}$ Thin Films with Excess Si. J. Appl. Phys. 2009, 106, 014305.

(17) Talbot, E.; Larde, R.; Pareige, P.; Khomenkova, L.; Hijazi, K.; Gourbilleau, F. Nanoscale Evidence of Erbium Clustering in Er-Doped Silicon-Rich Silica. Nanoscale Res. Lett. 2013, 8, 39.

(18) Choi, H.-J.; Shin, J. H.; Suh, K.; Seong, H.-K.; Han, H.-C.; Lee, J.-C. Self-Organized Growth of $\mathrm{Si} / \mathrm{Silica} / \mathrm{Er}_{2} \mathrm{Si}_{2} \mathrm{O}_{7}$ Core-Shell Nanowire Heterostructures and their Luminescence. Nano Lett. 2005, 5, 2432-2437.

(19) Choi, W. C.; Lee, H. N.; Kim, E. K.; Kim, Y.; Park, C.-Y.; Kim, H. S.; Lee, J. Y. Violet/Blue Light-Emitting Cerium Silicates. Appl. Phys. Lett. 1999, 75, 2389-2391.

(20) Miritello, M.; Lo Savio, R.; Iacona, F.; Franzò, G.; Irrera, A.; Piro, A. M.; Bongiorno, C.; Priolo, F. Efficient Luminescence and Energy Transfer in Erbium Silicate Thin Films. Adv. Mater. 2007, 19, $1582-1588$

(21) Masaki, K.; Isshiki, H.; Kawaguchi, T.; Kimura, T. The Effect of Annealing Conditions on the Crystallization of Er-Si-O Formed by Solid Phase Reaction. Opt. Mater. 2006, 28, 831-835. 
(22) Isshiki, H.; de Dood, M. J. A.; Polman, A.; Kimura, T. SelfAssembled Infrared-Luminescent Er-Si-O Crystallites on Silicon. Appl. Phys. Lett. 2004, 85, 4343-4345.

(23) Kepinski, L.; Hreniak, D.; Strek, W. Microstructure and Luminescence Properties of Nanocrystalline Cerium Silicates. J. Alloys Compd. 2002, 341, 203-207.

(24) Li, J.; Zalloum, O.; Roschuk, T.; Heng, C.; Wojcik, J.; Mascher, P. The Formation of Light Emitting Cerium Silicates in CeriumDoped Silicon Oxides. Appl. Phys. Lett. 2009, 94, 011112.

(25) Beainy, G.; Weimmerskirch-Aubatin, J.; Stoffel, M.; Vergnat, M.; Rinnert, H.; Castro, C.; Pareige, P.; Talbot, E. Structural and Optical Study of Ce Segregation in Ce-Doped $\mathrm{SiO}_{1.5}$ Thin Films. J. Appl. Phys. 2015, 118, 234308.

(26) Gault, B.; Moody, M. P.; Cairney, J. M.; Ringer, S. P. Atom Probe Microscopy; Springer-Verlag: New York, 2012.

(27) Thompson, K.; Lawrence, D.; Larson, D.; Olson, J.; Kelly, T.; Gorman, B. In Situ Site-Specific Specimen Preparation for Atom Probe Tomography. Ultramicroscopy 2007, 107, 131-139.

(28) Larson, D.; Foord, D.; Petford-Long, A.; Liew, H.; Blamire, M.; Cerezo, A.; Smith, G. Field-Ion Specimen Preparation Using Focused Ion-Beam Milling. Ultramicroscopy 1999, 79, 287-293.

(29) Kovalev, D.; Heckler, H.; Polisski, G.; Koch, F. Optical Properties of Si Nanocrystals. Phys. Status Solidi B 1999, 215, 871932.

(30) Iacona, F.; Bongiorno, C.; Spinella, C.; Boninelli, S.; Priolo, F. Formation and Evolution of Luminescent Si Nanoclusters Produced by Thermal Annealing of $\mathrm{SiO}_{x}$ Films. J. Appl. Phys. 2004, 95, 37233732 .

(31) Vurpillot, F.; Bostel, A.; Blavette, D. Trajectory Overlaps and Local Magnification in Three-Dimensional Atom Probe. Appl. Phys. Lett. 2000, 76, 3127-3129.

(32) Kepinski, L.; Wolcyrz, M.; Marchewka, M. Structure Evolution of Nanocrystalline $\mathrm{CeO}_{2}$ Supported on Silica: Effect of Temperature and Atmosphere. J. Solid State Chem. 2002, 168, 110-118.

(33) Weimmerskirch-Aubatin, J.; Stoffel, M.; Devaux, X.; Bouche, A.; Beainy, G.; Talbot, E.; Pareige, P.; Fagot-Revurat, Y.; Vergnat, M.; Rinnert, H. Observation of a Nanoscale Phase Separation in BlueEmitting Ce-Doped $\mathrm{SiO}_{1.5}$ Thin Films. J. Mater. Chem. C 2015, 3, 12499-12506.

(34) Roussel, M.; Talbot, E.; Pareige, C.; Nalini, R. P.; Gourbilleau, F.; Pareige, P. Confined Phase Separation in $\mathrm{SiO}_{\mathrm{X}}$ Nanometric Thin Layers. Appl. Phys. Lett. 2013, 103, 203109.

(35) Roussel, M.; Talbot, E.; Pareige, P.; Gourbilleau, F. Influence of the Supersaturation on Si Diffusion and Growth of Si Nanoparticles in Silicon-Rich Silica. J. Appl. Phys. 2013, 113, 063519.

(36) Gu, H.; Zheng, R.; Zhang, X.; Xu, B. Facile One-Pot Synthesis of Bifunctional Heterodimers of Nanoparticles: A Conjugate of Quantum Dot and Magnetic Nanoparticles. J. Am. Chem. Soc. 2004, 126, 5664-5665.

(37) Okubo, M.; Yamashita, T.; Minami, H.; Konishi, Y. Preparation of Micron-Sized Monodispersed Highly Monomer-"Adsorbed" Polymer Particles Having Snow-Man Shape by Utilizing the Dynamic Swelling Method with Tightly Cross-Linked Seed Particles. Colloid Polym. Sci. 1998, 276, 887-892. 